\title{
Electro-responsive polyelectrolyte-coated surfaces
}

\author{
V. Sénéchal, ${ }^{\mathrm{ab}} H$. Saadaoui, ${ }^{\mathrm{ab}}$ J. Rodriguez-Hernandez ${ }^{\mathrm{c}}$ \\ and C. Drummond*ab
}

The anchoring of polymer chains at solid surfaces is an efficient way to modify interfacial properties like the stability and rheology of colloidal dispersions, lubrication and biocompatibility. Polyelectrolytes are good candidates for the building of smart materials, as the polyion chain conformation can often be tuned by manipulation of different physico-chemical variables. However, achieving efficient and reversible control of this process represents an important technological challenge. In this regard, the application of an external electrical stimulus on polyelectrolytes seems to be a convenient control strategy, for several reasons. First, it is relatively easy to apply an electric field to the material with adequate spatiotemporal control. In addition, in contrast to chemically induced changes, the molecular response to a changing electric field occurs relatively quickly. If the system is properly designed, this response can then be used to control the magnitude of surface properties. In this work we discuss the effect of an external electric field on the adhesion and lubrication properties of several polyelectrolyte-coated surfaces. The influence of the applied field is investigated at different $\mathrm{pH}$ and salt conditions, as the polyelectrolyte conformation is sensitive to these variables. We show that it is possible to fine tune friction and adhesion using relatively low applied fields.

\section{Introduction}

The interface between a material and the surrounding environment determines many important properties. Wettability, adhesion and haptic perception are often controlled by the outmost molecular layers at the interface. Thus, tuning material properties often translates into controlling the conformation and/or organization of molecules at the interfacial level. Coating a particular material with stimuliresponsive species at the external boundary may confer conventional materials with responsive ('smart') properties.

${ }^{a}$ CNRS, Centre de Recherche Paul Pascal (CRPP), UPR 8641, F-33600 Pessac, France. E-mail: drummond@ crpp-bordeaux.cnrs.fr

${ }^{b}$ Université de Bordeaux, Centre de Recherche Paul Pascal, F-33600 Pessac, France

'Instituto de Ciencia y Tecnología de Polímeros, CSIC, Juan de la Cierva 3, 28006, Madrid, Spain 
A number of stimuli have been proposed to trigger a material response; temperature, $\mathrm{pH}$, salinity and electromagnetic radiation are some commonly used examples. ${ }^{\mathbf{1} 2}$ To achieve stimuli-responsiveness, properly formulated responsive moieties have to be incorporated into the material. Changes in molecular conformation or intermolecular association are triggered in response to a particular stimulus. These changes then translate to changing overall material properties. In the design of smart materials, two aspects are often desired: fast response to stimuli application and reversibility of the response.

The use of the aforementioned stimuli has important drawbacks. On the one hand, local control of the stimuli and responsive elements at submicrometer scales is extremely challenging. In addition, material switching times of seconds to several minutes are often observed, thus hampering applications that require fine control.

Many examples of materials sensitive to electric fields have also been reported. Electroresponsive materials are generally based on the control of coulombic interactions. As electric signals can be remotely generated and locally controlled with great accuracy, these conformational changes may allow precise spatiotemporal control of material properties. The shapes of electroactive polymers (EAP) are modified upon application of an electric field. When the response is directly triggered by coulombic forces (e.g. electrostrictive ${ }^{3}$ or liquid crystalline polymers $^{4}$ ) the material is classified as an electronic EAP. In contrast, if the response is due to a changing physicochemical environment (e.g. local pH or ionic strength changes) due to the applied field, the EAP is called an ionic EAP. Polyelectrolytes (PEs) are a prototypical example of the latter category. Polyelectrolytes can be classified as strong (quenched) or weak (annealed). In the first category, the amount of charges in the polyionic chain is not modified by the external conditions ( $\mathrm{pH}$ or salt concentration). In contrast, the ionization degree of annealed PE can be regulated by environmental variables. ${ }^{5}$ Polyacids or polybases are examples of this type of PE. As the macromolecular conformation is governed by the charge density, this type of PE undergoes dramatic changes in response to a changing environment. Both types of $\mathrm{PE}$ are susceptible to conformational changes in the presence of an applied field, because of electrostatic Coulomb forces. However, the applied field can also trigger the redistribution of charged species. As the charge density of annealed $\mathrm{PE}$ is a strong function of $\mathrm{pH}$ or salt concentration, its conformation can be radically modified by an externally applied field; a changing density of charges on the polymer chain will induce important transformations of polymer conformation. Thus, annealed PEs are interesting candidates for the design of 'smart' electroactive polymer materials. In this work we describe how polyelectrolyte-based coatings may be used to control surface properties (friction and adhesion) by the adequate application of external direct or alternating electric fields.

\section{Materials and methods}

\section{Materials}

Polymer synthesis. Polystyrene-block-poly(acrylic acid) (PS-b-PAA) block copolymers were prepared by atom transfer radical polymerization (ATRP) in two steps following previously reported procedures. ${ }^{6}$ The polystyrene macroinitiator was prepared first using phenylethyl bromide ( $\mathrm{PhEBr}$ ) as the initiator. tert-Butyl acrylate 
was employed as the monomer for the second polymerization step. Finally, hydrolysis of the tert-butyl groups under acidic conditions (trifluoroacetic acid) led to the amphiphilic PS- $b$-PAA block copolymer. The average molecular formula of the copolymer used in this study was $\mathrm{PS}_{36}-b$ - $\mathrm{PAA}_{125}$, as determined by ${ }^{1} \mathrm{H}-\mathrm{NMR}$.

\section{Methods}

Polymer coating. Polymer coatings were prepared by two methods: self-assembly or the Langmuir-Schaefer (L-S) method. In the self-assembly method, the surfaces to be coated (mica or polystyrene-coated silicon wafers) were immersed in solutions of $1 \mathrm{mg} \mathrm{mL}{ }^{-1} \mathrm{PS}_{36}-b-\mathrm{PAA}_{125}$ at $\mathrm{pH} 10$ (by adding $\mathrm{KOH}$ ). Water-insoluble PS blocks of the copolymers readily adsorb on the surfaces, while the PAA block remains in contact with water. This method is relatively easy and reproducible. However, the density of irreversibly adsorbed polymer cannot be readily controlled. In addition, large grafting polymer densities are out of reach because of the steric hindrance of adsorbed polymers on the later arriving molecules.

L-S polymer coatings were prepared as described by Currie and co-workers. ${ }^{7} \mathrm{~A}$ few drops of a $1 \mathrm{~g} \mathrm{~L}^{-1}$ solution of $\mathrm{PS}_{36}-b-\mathrm{PAA}_{125}$ in $60 \% / 40 \%$ dioxane/toluene were spread at the air-water interface on a Langmuir trough filled with $0.1 \mathrm{M} \mathrm{NaCl}$ in water. After solvent evaporation, the copolymer monolayers were compressed at different surface pressures, $\Pi$, and transferred to gold/PS-coated Si wafers. $50 \mathrm{~nm}$ gold layers were used as the electrodes for electroresponsive experiments. $50 \mathrm{~nm}$ thick PS layers were spin-coated on top of the metallic electrode to act as a primer layer, in order to improve the adherence of the copolymer coating. After transferring the copolymer, the surfaces were annealed at $110{ }^{\circ} \mathrm{C}$ for $10 \mathrm{~min}$ to improve the anchoring of the diblock copolymer molecules on the primer PS film. This method has been used in a number of studies related to the surface properties of PAA polymer brushes. ${ }^{8,9}$

Surface forces apparatus, SFA. The interaction forces between mica surfaces coated by self-assembled PE layers were measured using a home-built Surface Forces Apparatus. This technique has been abundantly described in the literature. ${ }^{10}$ Briefly, molecularly smooth mica surfaces glued onto cylindrical silica lenses (placed with their axes perpendicular to each other) are used to obtain a well-defined contact geometry. Multiple Beam Interferometry is used to measure the thickness and refractive index of the film confined between the surfaces, with an accuracy of $0.1 \mathrm{~nm}$ and 0.01 , respectively. ${ }^{11}$ For this purpose, reflective silver films are deposited on the backside of the mica surfaces. In this work we also used these silver layers as electrodes to apply an electric film through the confined lubricant film. ${ }^{12}$ The deformation of double cantilever springs is used to measure the normal and lateral forces between the mica surfaces. In a typical experiment the surfaces are brought to a certain separation, $D$, and a normal compressive load, $L$, may be applied. A lateral displacement between the surfaces is imposed at a certain velocity, $V$, by using piezoelectric actuators, and the force induced by this displacement is measured. As the range of surface displacement is limited, shearing cycles are typically applied. One of the surfaces is displaced at constant velocity, $V$, over a certain distance, and then the driving direction is reversed..$^{13}$

Atomic force microscopy, AFM. The morphologies of the $\mathrm{PS}_{36}-b-\mathrm{PAA}_{125}$ films in air were determined by Atomic Force Microscopy in tapping mode (Icon and Multimode, Bruker). 
Force versus separation curves were measured with $2 \mu \mathrm{m} \mathrm{SiO}{ }_{2}$ colloidal probes on $200 \mu \mathrm{m}$ long silicon nitride triangular cantilevers (sQUBE CP-PNPL-SiO-A-2). The manufacturer's nominal cantilever spring constant was $0.08 \mathrm{~N} \mathrm{~m}^{-1}$. The interaction forces and adhesion between the colloidal probe and the $\mathrm{PS}_{36}-b$ PAA $_{125}$ films were measured under different applied voltages between the gold substrate and the bulk solution. From the raw data (the measured photodiode voltage vs. scanner position), data for the cantilever deflection vs. the tipsubstrate relative distance were calculated, considering tip-substrate contact at the constant compliance region, as is frequently described in the literature. ${ }^{14}$

Quartz crystal microbalance with dissipation monitoring, QCM-D. Conformational changes of the $\mathrm{PS}_{36}-b$ - $\mathrm{PAA}_{125}$ monolayers were investigated using a commercial quartz crystal microbalance (QCM-D E1, Q-Sense). In this technique the resonance frequency, $f$, of a quartz resonator is measured; if the effective mass of the crystal changes due to material adsorption, a shift in the crystal resonance frequency, $\Delta f$, is observed. The damping of the oscillation of the crystal can also be measured and used to calculated the "dissipation factor", $D$ (the inverse of the quality factor of the resonance peak). ${ }^{15}$ In a QCM-D experiment, the measured $\Delta f$ and $\Delta D$ values can be related to the thickness and viscoelastic properties of the material adsorbed on the quartz crystal by using adequate models. ${ }^{15}$

We used 5.0 MHz quartz resonators with gold electrodes, which were coated with $50 \mathrm{~nm}$ of PS and a monolayer of $\mathrm{PS}_{36}-b-\mathrm{PAA}_{125}$. The effect of $\mathrm{pH}$ or salinity on polymer conformation was investigated by measuring the response of the odd harmonics $(n=3$ to $n=13)$. To analyse the measured data, we fitted the measured $\Delta f$ and $\Delta D$ values by using the QTM software, written by Dr Diethlem Johannsmann. ${ }^{16}$

\section{Results and discussion}

\section{Polymer coating morphology}

AFM micrographs of the copolymer adsorbed layers were measured under dry and wet conditions. The typical results are presented in Fig. 1. As can be observed, uniformly coated surfaces were obtained by both the L-S and self-assembly methods. As has been thoroughly reported in the literature, PS islands surrounded by a PAA corona are observed under dry conditions (Fig. 1a-c). ${ }^{9}$ In the L$\mathrm{S}$ method, the areal density of the islands increases with increasing applied surface pressure during transfer (Fig. 1a and b); a good control of the copolymer grafting density is achieved. As can be anticipated, the density obtained by the self-assembly method (Fig. 1c) is similar to that obtained by the L-S method at low pressures. The micrograph presented in Fig. 1d was measured under water, in the absence of the PS primer layer. There is also substantial copolymer adsorption in this case. However, the appearance of lines that are preferentially oriented in the slow scan direction (upward in the figure) suggests that the polymer molecules are not firmly attached to the substrate.

\section{Responsive polymer coating}

The degree of solvation and the hydrated size of the adsorbed PE layer vary with the environmental conditions. Changing the $\mathrm{pH}$ or ionic strength of the surrounding water causes important changes in polymer conformation. This can 

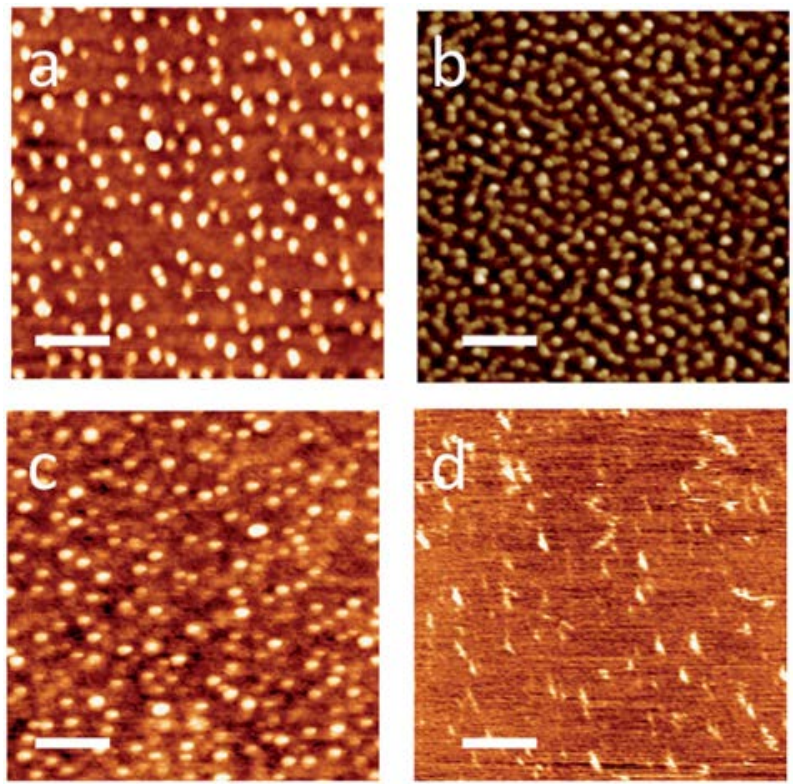

Fig. 1 AFM micrographs of $\mathrm{PS}_{36}-b-\mathrm{PAA}_{125}$ films deposited by (a) $\mathrm{L}-\mathrm{S}$ at $0.2 \mathrm{mN} \mathrm{m}^{-1}$; (b) $\mathrm{L}-$ $\mathrm{S}$ at $1 \mathrm{mN} \mathrm{m}{ }^{-1}$; (c) and (d) self-assembly at $\mathrm{pH} 10$ from a solution of $1 \mathrm{mg} \mathrm{mL}^{-1} \mathrm{PS}_{36}-b-$ PAA $_{125}$. (a)-(c) Adsorption on PS-coated gold surfaces. The micrographs were measured in air in tapping mode; (d) self-assembly on mica. The micrograph was measured in water in contact mode. The scale bars correspond to $200 \mathrm{~nm}$.

be readily evidenced by QCM-D. As explained above, with this technique, the changes in film thickness are estimated by measuring $\Delta f$. As in our tests the polymer brush was preadsorbed on the quartz crystal (before the QCM-D measurements), no absolute value of the polymer coating was measured. However, apparent shifts in film thickness, due to changes in the degree of polymer swelling in a changing environment, can be estimated. As can be observed in Fig. 2, a regular evolution of effective thickness is observed upon exposing the copolymer coated quartz crystals to different conditions of $\mathrm{pH}$ or salt concentration. For all the experiments discussed in this work, the value of $\mathrm{pH}$ has been adjusted by adding small amounts of $\mathrm{HCl}$ or $\mathrm{KOH}$ as required, right before the experiment. We have purposefully avoided using buffer solutions to minimize the number of ionic species present. PAA is a weak polyacid; at low $\mathrm{pH}$ values the acrylic acid monomers are protonated, while they get increasingly ionized at high pH. As can be observed in Fig. 2a, the increasing electrostatic repulsion between charged AA monomers at high $\mathrm{pH}$ causes an important increment in the effective size of the hydrated polymer coating. At large $\mathrm{pH}$ values the monomers are mostly ionized, and the polymer chain behaves as a quenched PE. A more complicated scenario is observed with increasing ionic strength; the addition of salt significantly modifies the polymer chain conformation. The effective thickness of the PE coating initially increases, reaching a maximum value, and then decreases with increasing salt concentration (Fig. 2b). The nonmonotonic behaviour of PAA brush thickness with ionic strength has been previously reported in the literature. ${ }^{17}$ At very low salt concentrations, the proton concentration close to the 

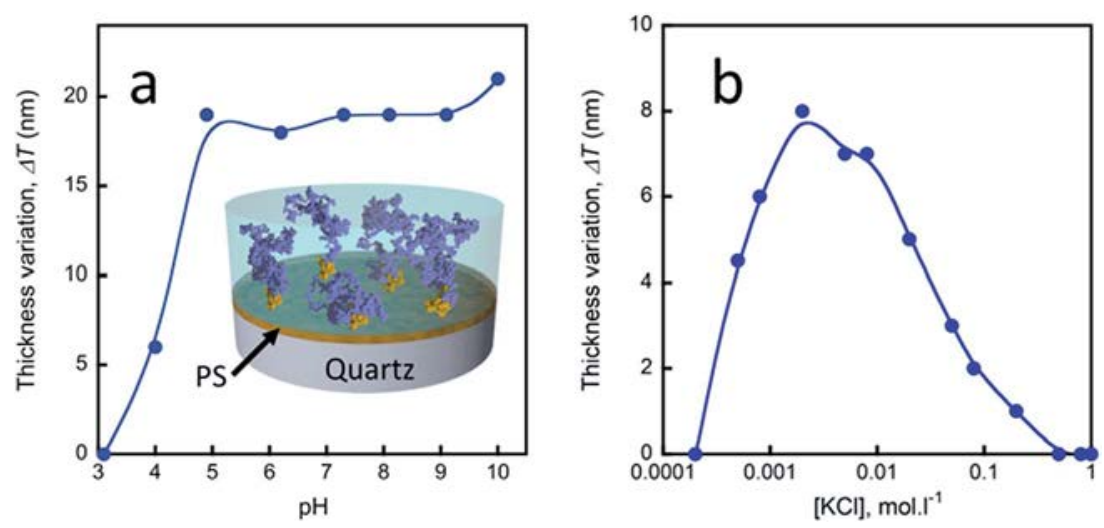

Fig. 2 QCM-D: variation in polymer film thickness, $\Delta T$, as a function of (a) $\mathrm{pH}$ and (b) $\mathrm{KCl}$ concentration, calculated from the shifts in resonance frequency and the energy dissipation of quartz crystals coated by a $\mathrm{PS}_{36}-b-\mathrm{PAA}_{125}$ monolayer deposited using the $\mathrm{L}-\mathrm{S}$ method at $\Pi=0.2 \mathrm{mN} \mathrm{m}^{-1}$. The results obtained for the odd harmonics, $n=3$ to $n=13$, were used to calculate $\Delta T$. Inset: schematic representation of the coated quartz crystal. Diblock copolymers are deposited on a $50 \mathrm{~nm}$ thick PS layer spin-coated on the goldcoated quartz crystal.

grafted chains is large due to electroneutrality, and the degree of dissociation of $\mathrm{PE}$ chains is reduced. Inter and intramolecular polymer interaction dominates, and the polymer chain collapses. Increasing salt concentration allows the exchange of protons by neutral counterions, thus progressively increasing the effective degree of PE dissociation. Therefore, the osmotic pressure due to the counterions near the polymer chains (because of electroneutrality) increasingly forces the swelling of the grafted chains (osmotic brush). At large salt concentrations the electrostatic repulsion between charged monomers is progressively screened, causing a decrease in polymer size (salted brush). A more detailed theoretical discussion of this progression can be found in the literature. ${ }^{\mathbf{1 8 - 2 0}}$

The changes in $\mathrm{PE}$ conformation with $\mathrm{pH}$ and ionic strength are reproducible and reversible. Many cycles of swelling-collapse of the grafted chains can be executed without a significant loss of response. Thus, the variation of $\mathrm{PE}$ conformation on the physicochemical environment can be used for the effective design of 'smart' materials. As shown in the following section the environment can be externally manipulated by the application of an external electric field, thus triggering changes in polymer conformation. If adequately designed, these changes have important implications for surface properties.

\section{Polymer coating interpenetration tuning: control of friction forces}

Neutral polymer layers immersed in a good solvent can act as very effective boundary lubricants between sliding surfaces. The ability of the polymer layers to sustain a substantial compressive load without extensive interpenetration of the opposite boundary layers - due to osmotic/entropic factors-is the reason for this remarkable property. ${ }^{21}$ Analogously, it has been reported that charged polyelectrolytes show extremely good lubrication properties. ${ }^{22,23}$ When two polyelectrolyte layers are subjected to compression, their hydration layers overlap and 
water in the interlayer region effectively acts as a low viscosity lubricating fluid. Reported theoretical models ${ }^{24,25}$ and molecular dynamic simulations ${ }^{26-28}$ support the idea that the lubrication quality is determined by the degree of interpenetration between opposite brushes under compression and shear. Thus, if the interpenetration between the polymer layers is forced either by higher compressive loads or worsening solvent conditions, a sharp deterioration of the lubrication properties is observed. Higher energy dissipation occurs when the interpenetrating polymer chains are stretched out under shear. Therefore, mastering the interpenetration between opposite polyelectrolyte lubricant layers provides a way to tune the friction forces. As we have shown in the past, an alternating electric field can be used for this purpose. ${ }^{12}$ As an example of this method, the variation of friction forces between mica surfaces coated by a self-assembled $\mathrm{PS}_{36}-b-\mathrm{PAA}_{125}$ lubricant layer due to the application of an alternating electric field is shown in Fig. 3. In this experiment the $\mathrm{pH}$ of the media was adjusted to 10.1 (by adding $\mathrm{KOH}$ ) to ensure complete ionization of the acrylic acid monomers. To avoid any temporal variation in the normal load, $L$, as a consequence of a changing Coulomb force, a square wave voltage is applied. As can be observed in the figure, the friction progressively decreases as the amplitude of the applied voltage is augmented. The rapid oscillatory perturbation of the polyions ensures that polymers on opposite surfaces pass each other, thus reducing shear-induced shear strain and energy dissipation. The transition between different friction states happened immediately after applying or removing the external field. In addition, no signs of transient (non-steady) states could be detected within our temporal resolution $(10 \mathrm{~Hz})$. As can be observed in Fig. 3, the high friction state was unchanged before and after the application of the field; it is apparent that no permanent modification of the lubricant layer was induced throughout the test. The method described here is related to the friction reduction observed when boundary lubricant layers under shear are perturbed by a smallamplitude normal oscillation. ${ }^{29}$ However, it is apparent that electric signals are simpler to produce and manipulate, thus offering superior possibilities for the fine spatiotemporal control of friction.

As we have reported before, ${ }^{12}$ the measured normal force between the confining surfaces appears to be unaltered during the process; no variation in the
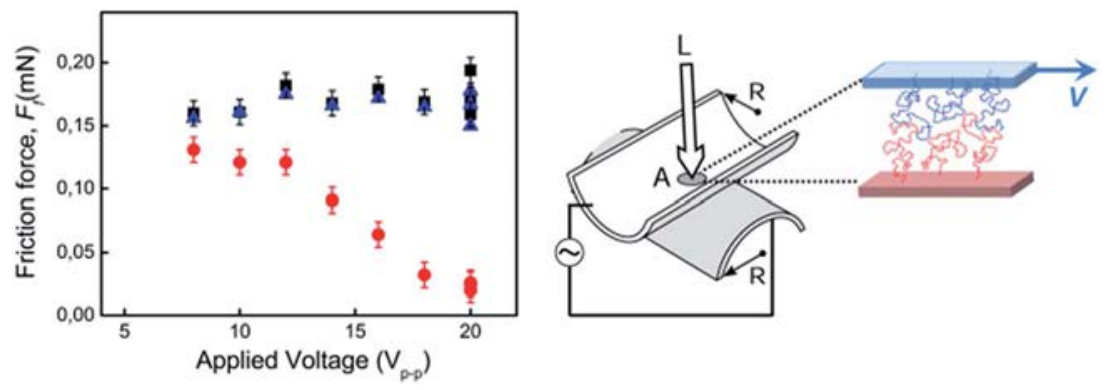

Fig. 3 Friction force between opposite mica surfaces coated by $\mathrm{PS}_{36}-b-\mathrm{PAA}_{125}$ deposited by self-assembly as a function of the externally applied square wave alternating voltage amplitude (red circles). $f=450 \mathrm{~Hz}, L=5.56 \mathrm{mN}, V=0.3 \mu \mathrm{m} \mathrm{s}^{-1}$. $F_{\mathrm{f}}$ is clearly larger and unchanged before (black squares) and after (blue triangles) application of the field. Right panel: Schematic illustration of the crossed-cylinder geometry used in the SFA and interpenetrating brushes under shear. 
magnitude or the range of the normal interaction is detected. This result suggests that the applied field fails to cause any dramatic conformational change in the adsorbed polymer chains. As the PE chains are mostly dissociated under the experimental conditions, and an important fraction of the potential drop in the multilayer system (mica-PE-mica) happens through the dielectric mica surfaces (the mica thickness is $4.018 \mu \mathrm{m}$ while the confined polymer film is of the order of $10 \mathrm{~nm}$ in this particular experiment), this result is by no means surprising; the applied external field is not large enough to trigger a large conformational change in the polymer. Nevertheless, it is apparent that a small amplitude oscillatory motion of the opposite PE chains is enough to substantially decrease the interpenetration between the chains of the opposite brushes, shear-induced deformation and energy dissipation under shear.

These results show how small variations in PE conformation, obtained at relatively small applied voltages, may have significant consequences on material properties. If the system is properly designed, useful material responsiveness can be achieved even in the absence of a dramatic molecular response. In the next example we show how more important PE conformational changes can be induced by applying an external field if the environmental conditions are properly selected.

\section{Polymer coating conformational tuning: adhesion control}

At basic pH, PAA chains are completely dissociated and behave as strong PEs for all practical purposes. Dramatic conformational changes are probably out of reach under these conditions. On the contrary, if the $\mathrm{pH}$ is reduced closer to the $\mathrm{p} K_{\mathrm{a}}$ of the polymer, important conformational changes can be triggered by small changes in the external environment. For instance, as shown by the QCM-D results ( $c f$. Fig. 2a), the apparent thickness of the polymer coating significantly increases when the $\mathrm{pH}$ is changed from 4 to 5 . This conformational change will have important consequences on the properties of the layer, and in particular on its interaction with other entities (e.g. colloidal aggregates, nanoparticles, viruses...), modifying properties like surface adhesion and wettability.

Therefore, by changing the local environment close to the polymer, molecular conformational changes can be triggered and surface properties can be externally tuned with a good degree of control. With this aim, we have investigated the effect of an externally applied electric bias on the interaction between a silica colloidal probe and a $\mathrm{PS}_{36}-b-\mathrm{PAA}_{125}$ coated substrate, using AFM as described in the experimental section. The typical results of the interaction force profiles obtained are presented in Fig. 4; as can be observed in the figure, the applied electric field has implications at different levels. Long range electrostatic repulsion can be observed for separations, $D$, larger than $10 \mathrm{~nm}$. The silica colloidal probe is negatively charged in water at $\mathrm{pH} 4.5$, thus a long range electrostatic repulsive force can be anticipated when it interacts with a negatively charged surface. As can be expected, this force is reduced by applying a positive bias to the substrate (Fig. 4a and c), and is substantially larger when a negative bias is applied (Fig. 4b and d). Similar results have been discussed by other groups in the past; ${ }^{30-32}$ however, it is very informative to consider the characteristic decay length of the long-range repulsive force, $\lambda$, which can be estimated by fitting the deflection $v s$. separation data for separations larger than $c a .7 \mathrm{~nm}$ to a single-exponential decay. 

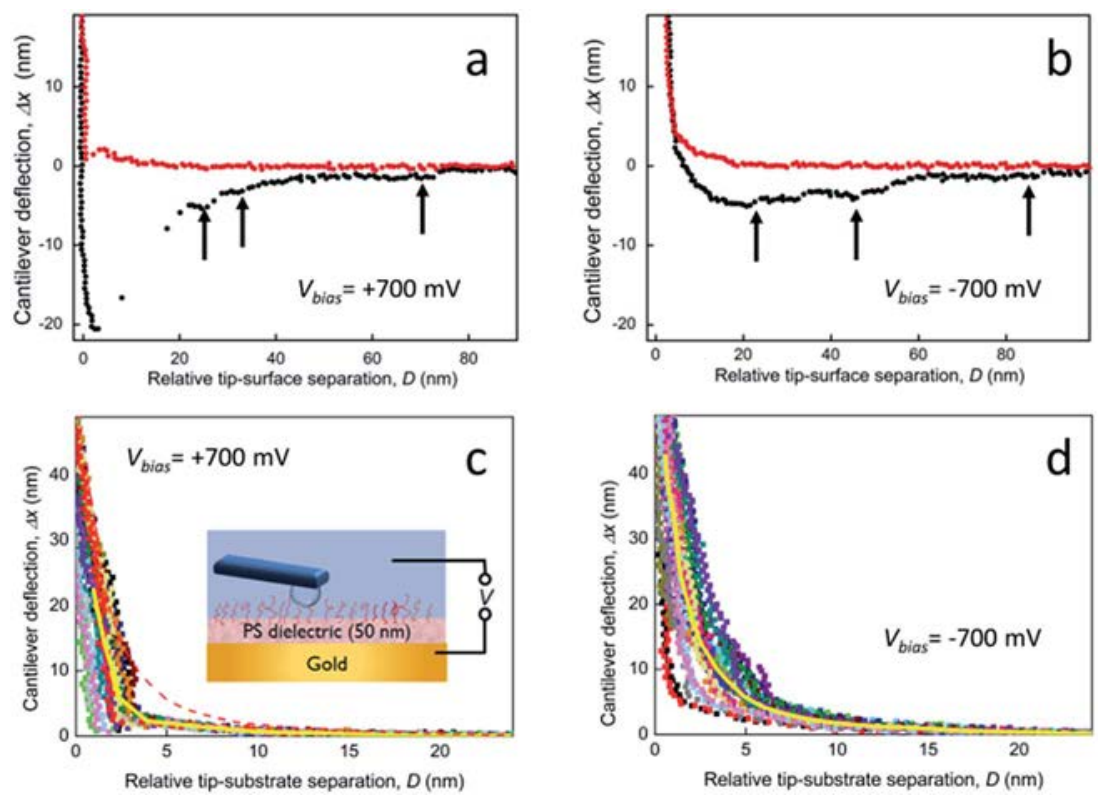

Fig. 4 Normal interaction force measured by AFM between a silica colloidal probe and a $\mathrm{PS}_{36}-b-\mathrm{PAA}_{125}$ monolayer deposited by the $\mathrm{L}-\mathrm{S}$ method at $\Pi=0.2 \mathrm{mN} \mathrm{m}{ }^{-1}$. $\mathrm{pH}=4.5$, adjusted by addition of $\mathrm{HCl},[\mathrm{KCl}]=2 \mathrm{mM}$. (a) and (b) show the typical approach (red) and separation (black) curves. (c) and (d) show the 20 different approach curves measured in different positions on the substrate to illustrate the variability in the measured interaction. The continuous yellow lines correspond to the arithmetic mean of the measured profiles. The mean curve in (d) is reproduced as a red dashed line in (c) to simplify the comparison between the two data sets. An electric potential bias of $700 \mathrm{mV}$ for (a) and (c) and $-700 \mathrm{mV}$ for (b) and (d) was applied between the gold electrode and the bulk aqueous solution. Inset: representation of the experimental setup.

In the absence of an externally applied field, or with applied voltages in the range of $-500 \mathrm{mV}$ to $1 \mathrm{~V}, \lambda$ agrees very well with the Debye length, $\lambda_{\mathrm{D}}$, expected for the ionic strength of the aqueous solution ${ }^{33}\left(\lambda_{\mathrm{D}} c a .6 .8 \mathrm{~nm}\right.$ for the $2 \mathrm{mM} \mathrm{KCl}$ concentration used). In contrast, for larger negative applied voltages (in absolute value), $\lambda$ increases to $c a .9 \mathrm{~nm}$; this is clearly in conflict with a pure electrostatic repulsion, and suggests a steric contribution to the repulsive force due to a changing conformation of the polymer coating. This conformational change is confirmed by the shorter-range steric $(D<10 \mathrm{~nm})$ repulsive force, which is a consequence of the compression of the polymer coating by the colloidal probe, and is substantially altered by the applied field. By comparing the results presented in Fig. $4 \mathrm{c}$ and d, it is apparent that the range of the repulsive steric force is much larger for the negatively applied bias, compared with the range for the positive one. Even though there is uncertainty regarding the absolute tipsubstrate distance and the position of the zero-distance value (intrinsic to the AFM technique for measuring forces), it seems clear that a conformational transition is being induced by the applied voltage. This change in the polymer layer has important consequences on the adhesive force between the tip and the substrate, as can be concluded by comparing the separation curves (Fig. 4a and b); 
the maximum attractive force is substantially reduced when the negative bias is applied. While a clear adhesive jump-out due to the attractive dispersion forces is observed when the positive voltage is applied (Fig. 4a), a long range small constant attractive force is observed for the negative bias (Fig. 4b). This difference clearly shows that a more intimate probe-substrate contact is achieved when the positive bias is applied. A number of steps (signalled by arrows in the figures) and a negative plateau are also observed in the retraction curves. These features have been observed in the past in force studies of adsorbed macromolecules, and have been associated with the progressive detachment of polymer segments adsorbed on the colloidal probe. ${ }^{34,35}$ These detachment events are absent if the absolute value of the negative bias is sufficiently large ( $c f$. Fig. 5).

As mentioned above, the changes in tip-substrate interaction forces reported here go beyond the variation in electrostatic interaction due to the applied field, which has been reported by other groups in the past; ${ }^{30,32}$ an important change in polymer conformation appears as a consequence of the applied field. Good control of the tip-substrate adhesion can be obtained with the applied voltage, as can be observed in Fig. 5. This figure shows the mean maximum adhesive force observed upon retraction (the minimum of the retraction deflection curve) for the different biases applied. At positive (or small negative) bias the adhesion appears to be mostly unchanged. In contrast, a progressive reduction of adhesion is observed when increasingly larger negative biases are applied. Interestingly, at a sufficiently large negative tension, the tip-substrate adhesive force completely disappears, even when large compressive forces were applied. The voltageinduced enhanced steric repulsive force greatly exceeds the tip-substrate dispersive attractive interaction.

As the electric field can be remotely controlled with high precision, this result suggests that fine spatiotemporal control of adhesion can be achieved by this method. Strategies of surface functionalization for the active control of surface

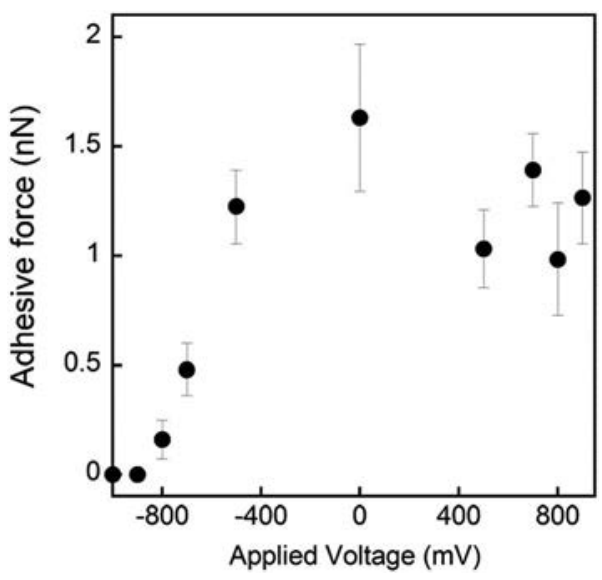

Fig. 5 Mean adhesion between a silica colloidal probe and a $\mathrm{PS}_{36}-b-\mathrm{PAA}_{125}$ monolayer deposited by the $L-S$ method at $\Pi=0.2 \mathrm{mN} \mathrm{m}^{-1}$ as a function of the applied bias between the gold electrode and the aqueous environment. The reported values are the maximum cantilever forces in retraction force curves calculated using the manufacturer's nominal spring constant of the cantilever. $\mathrm{pH}=4.5$ and $[\mathrm{KCl}]=2 \mathrm{mM}$. 
properties using externally applied electric fields have been proposed in the past. These methods, based on self-assembled monolayers (SAMs) with well-controlled molecular compositions, allow a good digital (on-off) control of adhesion. ${ }^{36-38}$ In contrast, the methods explored in this work, based on the continuous conformational transition of a necessarily polydisperse polyelectrolyte coating, make a more versatile analogue (continuous) control of surface properties possible, as can be observed in Fig. 3 and 5 .

It is important to identify the driving force responsible for the observed polymer conformational change. We believe that the externally applied field stretches the ionic bonding between the protons and the polyion, thus weakening it. This translates to an effective increase of the degree of dissociation of the polyion, which triggers the conformational change. In that sense, the reported phenomena can be understood along the lines of the dissociation field effect (second Wien effect); the dissociation constants of weak acids are enhanced upon application of sufficiently large electric fields. ${ }^{39}$ Thus, important conformational changes are observed if the environmental $\mathrm{pH}$ is close enough to the $\mathrm{p} K_{\mathrm{a}}$ of the polyacid. The effect described here is determined by the polyion conformation, and it depends non-trivially on the conditions of the $\mathrm{pH}$ and ionic strength of the media, and has important implications for other surface properties. A complete description of these effects will be reported in the near future.

\section{Conclusions}

Two examples illustrating how the properties of weak polyelectrolyte coatings can be manipulated by externally applied electric fields have been discussed. First, the control of polyelectrolyte lubrication by the application of an alternating field without significant polymer conformational changes was demonstrated. In the second example, we have shown how important polymer conformational changes can be triggered by the application of a small DC bias voltage, with important implications on interaction forces and adhesion. As electric fields can be remotely applied and controlled, these methods may open pathways for the fine spatiotemporal control of friction and adhesion in practical applications.

\section{Acknowledgements}

The authors acknowledge the financial support from the Ministerio de Economía y Competitividad (MINECO) project MAT2016-78437-R (Spain) and COST Action MP1303: Understanding and Controlling Nano and Mesoscale Friction.

\section{Notes and references}

1 M. A. C. Stuart, W. T. S. Huck, J. Genzer, M. Müller, C. Ober, M. Stamm, G. B. Sukhorukov, I. Szleifer, V. V. Tsukruk, M. Urban, F. Winnik, S. Zauscher, I. Luzinov and S. Minko, Nat. Mater., 2010, 9, 101-113.

2 T. P. Russell, Science, 2002, 297, 964-967.

3 M. Zhenyi, J. I. Scheinbeim, J. W. Lee and B. A. Newman, J. Polym. Sci., Part B: Polym. Phys., 1994, 32, 2721-2731.

4 W. Lehmann, H. Skupin, C. Tolksdorf, E. Gebhard, R. Zentel, P. Krüger, M. Lösche and F. Kremer, Nature, 2001, 410, 447-450. 
5 E. Raphael and J.-F. Joanny, Europhys. Lett., 1990, 13, 623-628.

6 A. Bousquet, E. Ibarboure, F. J. Teran, L. Ruiz, M. T. Garay, J. M. Laza, J. L. Vilas, E. Papon and J. Rodríguez-Hernández, J. Polym. Sci., Part A: Polym. Chem., 2010, 48, 2982-2990.

7 E. P. K. Currie, A. B. Sieval, G. J. Fleer and M. A. C. Stuart, Langmuir, 2000, 16, 8324-8333.

8 B. Liberelle and S. Giasson, Langmuir, 2008, 24, 1550-1559.

9 O. Theodoly, A. Checco and P. Muller, EPL, 2010, 90, 28004.

10 J. N. Israelachvili and P. M. McGuiggan, J. Mater. Res., 1990, 5, 2223-2231.

11 J. N. Israelachvili and S. Physics, J. Colloid Interface Sci., 1973, 44, 259-272.

12 C. Drummond, Phys. Rev. Lett., 2012, 109, 154302.

13 G. Luengo, F.-J. Schmitt, R. Hill and J. Israelachvili, Macromolecules, 1997, 30, 2482-2494.

14 H.-J. Butt, B. Cappella and M. Kappl, Surf. Sci. Rep., 2005, 59, 1-152.

15 I. Reviakine, D. Johannsmann and R. P. Richter, Anal. Chem., 2011, 83, 88388848.

16 QTM software is freely available for download at http://www.pc.tuclausthal.de/en/research/qcm-modelling/.

17 T. Wu, P. Gong, I. Szleifer, P. Vlček, V. Šubr and J. Genzer, Macromolecules, 2007, 40, 8756-8764.

18 E. B. Zhulina and O. V. Borisov, Langmuir, 2011, 27, 10615-10633.

19 M. Ballauff and O. Borisov, Curr. Opin. Colloid Interface Sci., 2006, 11, 316-323.

20 E. B. Zhulina, T. M. Birshtein and O. V. Borisov, Macromolecules, 1995, 28, 1491-1499.

21 J. Klein, E. Kumacheva, D. Mahalu, D. Perahia and L. J. Fetters, Nature, 1994, 370, 634-636.

22 U. Raviv, S. Giasson, N. Kampf, J.-F. Gohy, R. Jérôme and J. Klein, Nature, 2003, 425, 163-165.

23 C. Drummond, J. Rodríguez-Hernández, S. Lecommandoux and P. Richetti, J. Chem. Phys., 2007, 126, 184906.

24 J. B. Sokoloff, J. Chem. Phys., 2008, 129, 14901.

25 E. B. Zhulina and M. Rubinstein, Macromolecules, 2014, 47, 5825-5838.

26 G. S. Grest, Phys. Rev. Lett., 1996, 76, 4979-4982.

27 T. Kreer, M. H. Müser, K. Binder and J. Klein, Langmuir, 2001, 17, 7804-7813.

28 M. Sirchabesan and S. Giasson, Langmuir, 2007, 23, 9713-9721.

29 M. Heuberger, C. Drummond and J. Israelachvili, J. Phys. Chem. B, 1998, 102, 5038-5041.

30 V. Kuznetsov and G. Papastavrou, J. Phys. Chem. C, 2014, 118, 2673-2685.

31 S. Rentsch, H. Siegenthaler and G. Papastavrou, Langmuir, 2007, 23, 90839091.

32 S. D. Campbell and A. C. Hillier, Langmuir, 1999, 15, 891-899.

33 J. N. Israelachvili, Intermolecular and Surface Forces, Academic Press, 3rd edn, 2011.

34 M. Seitz, C. Friedsam, W. Jöstl, T. Hugel and H. E. Gaub, ChemPhysChem, 2003, 4, 986-990.

35 L. Sonnenberg, J. Parvole, O. Borisov, L. Billon, H. E. Gaub and M. Seitz, Macromolecules, 2006, 39, 281-288.

36 J. Lahann, S. Mitragotri, T.-N. Tran, H. Kaido, J. Sundaram, I. S. Choi, S. Hoffer, G. A. Somorjai and R. Langer, Science, 2003, 299, 371-374. 
37 M. Erdmann, R. David, A. Fornof and H. E. Gaub, Nat. Nanotechnol., 2010, 5, 154-159.

38 C. C. A. Ng, A. Magenau, S. H. Ngalim, S. Ciampi, M. Chockalingham, J. B. Harper, K. Gaus and J. J. Gooding, Angew. Chem., Int. Ed., 2012, 51, 7706-7710.

39 L. Onsager, J. Chem. Phys., 1934, 2, 599-615. 